\title{
Chaos, Mixing, Weakly Mixing and Exactness
}

\author{
Mohammed Nokhas Murad Kaki \\ Department of Mathematics, College of Science, University of Sulaimani, Sulaimani, Iraq \\ Email: moradkakaee@yahoo.com
}

How to cite this paper: Kaki, M.N.M. (2018) Chaos, Mixing, Weakly Mixing and Exactness. Open Access Library Journal, 5: e3773.

https://doi.org/10.4236/oalib.1103773

Received: June 27, 2017

Accepted: June 22, 2018

Published: June 26, 2018

Copyright (c) 2018 by author and Open Access Library Inc.

This work is licensed under the Creative Commons Attribution International License (CC BY 4.0).

http://creativecommons.org/licenses/by/4.0/

\begin{abstract}
In this paper, new definitions of chaos, exact chaos, mixing chaos, and weak mixing chaos called $\theta$-chaos, $\theta$ exact chaos, $\theta$-mixing chaos are introduced and extended to topological spaces. Our purpose is to investigate other types of transitivity, chaos and mixing, because when we confirm not existence of $\theta$-transitivity we confirm not existence of other types of transitive functions and its absence cannot find other types of transitivity. So the author must confirm the existence of this type of transitivity. We have proved that these chaotic definitions are all preserved under $\theta \mathrm{r}$-conjugation.
\end{abstract}

\section{Subject Areas}

Topological Dynamics

\section{Keywords}

$\theta$-Chaotic Maps, $\theta$-Mixing, Topological Exactness, Weak Mixing

\section{Introduction}

In this research paper, new types of minimal systems, transitivity and exactness are introduced and studied. This is intended as a survey article on transitivity and chaoticity of a discrete system given by $\theta$-irresolute self-map of a topological space. On one hand, it introduces postgraduate students to the study of new types of exactness, minimal systems and chaotic maps and gives an overview of results on the topic, but, on the other hand, it covers some of the recent developments of dynamics, technology, electronic and computer science. We denote the interior and the closure of a subset $A$ of $X$ by $\operatorname{Int}(A)$ and $C l(A)$ respectively. By a space $X$, we mean a topological space $(X, \tau)$. A point $x \in X$ is called a $\theta$-adherent point of $A$ [1], if $A \cap C l(U) \neq \phi$ for every open set $U$ containing $x$. The set of all $\theta$-adherent points of a subset $A$ of $X$ is called the $\theta$-closure of $A$ and is denoted by $C l_{\theta}(A)$. A subset $A$ of $X$ is called $\theta$-closed if 
$A=C l_{\theta}(A)$. Dontchev and Maki [2] have shown that if $A$ and $B$ are subsets of a space $X$, then $C l_{\theta}(A \cup B)=C l_{\theta}(A) \cup C l_{\theta}(B)$ and that

$C l_{\theta}(A \cap B)=C l_{\theta}(A) \cap C l_{\theta}(B)$. Recall that a space $(X, \tau)$ is Hausdorff if and only if every compact set is $\theta$-closed. The complement of a $\theta$-closed set is called a $\theta$-open set. The family of all $\theta$-open sets forms a topology on $X$ and is denoted by $\tau^{\theta}$. This topology is coarser than $\tau$ and that a space $(X, \tau)$ is regular if and only if $\tau=\tau^{\theta}$ [3]. Note also that the $\theta$-closure of a given set needs not be a $\theta$-closed set. Our purpose is to investigate some new types of transitivity, because when we confirm not existence of $\theta$-transitivity we can't confirm the existence of other types of transitive functions and then we can't study chaos theory, i.e. theta-transitivity absence cannot find other types of transitive maps therefore cannot find chaotic maps. For more knowledge about transitivity and chaotic maps see references [4] and [5].

\section{Preliminaries and Definitions}

\section{Definition 2.1}

A point $x \in X$ is said to be $A \theta$-interior point of $A$, if there exists an open set $\mathrm{U}$ containing $\mathrm{x}$ such that $U \subset \mathrm{Cl}(U) \subset A$.

The set of all $\theta$-interior points of $A$ is said to be the $\theta$-interior of $A$, and is denoted by $\operatorname{Int}_{\theta}(A)$. It is obvious that an open set $U$ in $X$ is $\theta$-open if $\operatorname{Int}_{\theta}(A)=U$.

\section{Definition 2.2}

1) A map $h: X \rightarrow Y$ is a homeomorphism if it is continuous, bijective and has a continuous inverse.

2) A map $h: X \rightarrow Y$ is $\theta$ r-homeomorphism if it is bijective and thus invertible and both $h$ and $h^{-1}$ are $\theta$-irresolute.

3) The systems $f: X \rightarrow X$ and $g: Y \rightarrow Y$ are topologically conjugate or conjugate if there is a homeomorphism $h: X \rightarrow Y$ such that $h \circ f=g \circ h$

4) The systems $f: X \rightarrow X$ and $g: Y \rightarrow Y$ are topologically $\theta$ r conjugate or $\theta$ conjugate if there is $\theta$ r-homeomorphism $h: X \rightarrow Y$ such that $h \circ f=g \circ h$

Definition 2.3 A map $f$ is said to be transitive (resp., $\theta$-transitive [6]) if for any non-empty open (resp., $\theta$-open) sets $U$ and $V$ in $X$, there exists $n \in \mathbf{N}$ such that $f^{n}(U) \cap V \neq \phi$.

Kaki definition 2.4 A map $f$ is said to be 1-transitive, if for every $x, y \in X$, there exists $n \in \mathbf{N}$ such that $f^{n}(x)=y$.

Theorem 2.5 every 1-transitive implies transitive.

\section{Proof:}

We have to prove that for any non-empty open sets $U$ and $V$ in $X$, there exists $n \in \mathbf{N}$ such that $f^{n}(U) \cap V \neq \phi$. Now, since $U$ and $V$ are non-empty so there is $x \in U$ and $y \in V$ so there is $n \in \mathbf{N}$ such that $f^{n}(x)=y$ since $f$ is 1-transitive and $y=f^{n}(x) \in f^{n}(U)$ but $y \in V$, we have $y \in f^{n}(U) \cap V$ i.e. $f^{n}(U) \cap V \neq \phi$.

\section{Definition 2.6}


The points $\mathrm{x}$ is called a non-wandering point if for every open set $U$ containing $\mathrm{x}$ there is an integer $n>0$ such that $f^{n}(U) \cap U \neq \phi$.

\section{Definition 2.7}

The non-wandering set of a map $f, N W(f)$, includes the points $\mathrm{x}$ such that for every open set $U$ containing $\mathrm{x}$ there is an integer $n>0$ such that $f^{n}(U) \cap U \neq \phi$.

\section{Definition 2.8}

The points $\mathrm{x}$ is called theta-non-wandering point if for every $\theta$-open set $U$ containing $\mathrm{x}$ there is an integer $n>0$ such that $f^{n}(U) \cap U \neq \phi$.

\section{Definition 2.9}

The theta-non-wandering set of a map $f, N W_{\theta}(f)$, includes the points x such that for every $\theta$-open set $U$ containing $\mathrm{x}$ there is an integer $n>0$ such that $f^{n}(U) \cap U \neq \phi$.

Proposition 2.10 Every non-wandering point is a theta-nonwandering point but not conversely.

\section{Action of a Group on a Topological Space}

If $G$ is a group and $X$ is a topological space, then a group action $\varphi$ of $G$ on $X$ is a function $\varphi: G \times X \rightarrow X$ such that $\varphi(g, x)=\varphi_{g}(x)$ that satisfies the following three axioms [7] and [8]:

1) $\varphi_{g}$ is continuous, for all $g$ in $G$

2) Identity: $\varphi_{g}(x)=x$ for all $x$ in $X$. (Here, $e$ denotes the neutral element of the group $G$.)

3) Compatibility $\varphi_{g h}(x)=\varphi_{g}\left(\varphi_{h}(x)\right)$ for all $g, h$ in $G$ and all $x$ in $X$.

The group $G$ is said to act on $X$ (on the left). The set $X$ is called a (left) $G$-set.

\section{Definition 3.1}

1) The action of $G$ on $X$ is called 1-transitive if $X$ is non-empty and if for each pair $x, y$ in $X$ there exists a $g$ in $G$ such that $\varphi_{g}(x)=y$.

2) The action of $G$ on $X$ is called topologically-transitive if $X$ is non-empty and if for each non-empty pair $U, V \subset X$, there exists a $g$ in $G$ such that $\varphi_{g}(U) \cap V \neq \phi$.

Theorem 3.2 Every 1-transitive implies topologically-transitive.

Proof: The same technique of theorem 2.5.

Definition 3.3 The action of $G$ on $X$ is called n-transitive if $X$ is non-empty and if for any two ordered sets of $n$ different points $\left\{x_{1}, \cdots, x_{n}\right\}$ and $\left\{y_{1}, \cdots, y_{n}\right\}$ in $X$ there exists a $g$ in $G$ such that $\varphi_{g}\left(x_{i}\right)=y_{i}$ for $i=1, \cdots, n$.

Definition 3.4 The action of $G$ on $X$ is called topologically n-transitive if $X$ is non-empty and if for each non-empty pair $U_{i}, V_{i} \subset X$, such that $U_{i} \cap U_{j}=\phi$ and $V_{i} \cap V_{j}=\phi$ for $i \neq j$ there exists a $g$ in $G$ such that $\varphi_{g}\left(U_{i}\right) \cap V_{i} \neq \phi$ for $i=1, \cdots, n$.

Definition 3.5 Relative to $\phi_{g}$ a point $x \in X$ is called wandering if there is an open $U$ containing x such that $\phi_{g}(U) \cap U=\phi$ for all $g \in G$.

\section{Definition 3.6}


1) A point $x \in X$ is $\theta$-recurrent if, for every $\theta$-open set $U$ containing $x$, infinitely many $n \in \mathbf{N}$ satisfy $g^{n}(x) \in U$.

2) Let $X$ be a topological space, $g: X \rightarrow X$ be $\theta$-irresolute map, then the function $g$ is called topologically $\theta$-strongly mixing if, given any nonempty $\theta$-open subsets $U, V \subseteq X \exists N \geq 1$ such that $g^{n}(U) \cap V \neq \phi$ for all $n>N$.

3) A subset $B$ of $X$ is g-invariant if $g(B) \subset B$. A non-empty $\theta$-closed invariant subset $B$ of $X$ is $\theta$-minimal, if $C l_{\theta}\left(O_{g}(x)\right)=B$ for every $x \in B$. A point $x \in X$ is $\theta$-minimal if it is contained in some $\theta$-minimal subset of $X$

Theorem 3.7 if $\mathrm{g}$ is topologically $\theta$-strongly mixing then it is also $\theta$-transitive but not conversely.

\section{Definition 3.8}

1) The function $f$ is $\theta$-exact if, for every nonempty $\theta$-open set $U$, there exists some $n \in \mathbf{N}$ such that $g^{n}(U)=X$.

2) The function $g$ is (topological) $\theta$-transitive (resp., $\theta$-mixing) if for any two nonempty $\theta$-open sets $U, V \subset X$, there exists some $n \in \mathbf{N}$ such that $g^{n}(U) \cap V \neq \phi$. (resp. $g^{m}(U) \cap V \neq \phi$ for all $m \geq n$ ).

3) The function $g$ is weak $\theta$-mixing if $g \times g$ is $\theta$-transitive on $X \times X$

4) The $\theta$-mixing function $g: X \rightarrow X$ is pure $\theta$-mixing if and only if there exists $\theta$-open set $U \subset X$ such that $g^{n}(U) \neq X$.

5) The function $g: X \rightarrow X$ is $\theta$-chaotic if $\mathrm{g}$ is $\theta$-transitive on $X$ and the set of periodic points of $g$ is $\theta$-dense in $X$.

6) The function $\mathrm{g}$ is called $\theta$-exact chaos (resp., $\theta$-mixing chaos and weakly $\theta$-mixing chaos) if $\mathrm{g}$ is $\theta$-exact (resp., $\theta$-mixing and weakly $\theta$-mixing) and $\theta$-chaotic function on the space $\mathrm{X}$.

Theorem 3.9 Theorem 3.9 topological $\theta$-exactness implies $\theta$-mixing implies weakly $\theta$-mixing implies $\theta$-transitivity

Theorem 3.10 If $f: X \rightarrow X$ and $g: Y \rightarrow Y$ are topologically $\theta$ r-conjugate by a $\theta$-homeomorphism $h: X \rightarrow Y$. Then

1) The map $f$ is $\theta$-exact if and only if $g$ is $\theta$-exact

2) The map $f$ is $\theta$-mixing if and only if $g$ is $\theta$-mixing

3) The map $f$ is $\theta$-chaotic if and only if $g$ is $\theta$-chaotic

4) The map $f$ is weakly $\theta$-mixing if and only if $g$ is weakly $\theta$-mixing.

Theorem 3.11 if $f: X \rightarrow X$ and $g: Y \rightarrow Y$ are $\theta r$-conjugate via $h: X \rightarrow Y$. Then

1) $T$ is $\theta$ transitive subset of $X \Leftrightarrow h(T)$ is $\theta$-type transitive subset of $Y$;

2) $T$ is $\theta$-mixing subset of $X \Leftrightarrow h(T)$ is $\theta$-mixing subset of $Y$.

Corollary A subset $B$ in the space $X$ is a chaotic set $\Leftrightarrow h(B)$ is a chaotic set in the space $Y$.

Proof (1)

Assume that $f: X \rightarrow X$ and $g: Y \rightarrow Y$ are topological systems which are topologically $\theta r$-conjugated by $h: X \rightarrow Y$. Thus, $h$ is $\theta$-homeomorphism (that is, $h$ is bijective and thus invertible and both $h$ and $h^{-1}$ are $\theta$-irresolute) and $h \circ f=g \circ h$ 
Suppose $T$ is $\theta$-type transitive subset of $X$. Let $A, B$ be $\theta$-open subsets of $Y$ with $B \cap h(T) \neq \phi$ and $A \cap h(T) \neq \phi$ (to show $g^{n}(A) \cap B \neq \varphi$ for some $n>0) . U=h^{-1}(A)$ and $V=h^{-1}(B)$ are $\theta$-open subsets of $X$ since $h$ is an $\theta$-irresolute.

Then there exists some $n>0$ such that $f^{n}(U) \cap V \neq \varphi$ since $T$ is $\theta$-type transitive subset of $X$, with $U \cap T \neq \phi$ and $V \cap T \neq \phi$. Thus (as $f \circ h^{-1}=h^{-1} \circ g$ implies $\left.f^{n} \circ h^{-1}=h^{-1} \circ g^{n}\right)$.

$\phi \neq f^{n}\left(h^{-1}(A)\right) \cap h^{-1}(B)=h^{-1}\left(g^{n}(A)\right) \cap h^{-1}(B)$

Therefore, $h^{-1}\left(g^{n}(A) \cap B\right) \neq \phi$ implies $g^{n}(A) \cap B \neq \phi$ since $h^{-1}$ invertible. So $h(T)$ is $\theta$-type transitive subset of $Y$.

\section{Proof (2)}

We only prove that if $T$ is topologically $\theta$-mixing subset of $Y$ then $h^{-1}(T)$ is also topologically $\theta$-mixing subset of $X$. Let $U, V$ be two $\theta$-open subsets of $X$ with $U \cap h^{-1}(T) \neq \phi$ and $V \cap h^{-1}(T) \neq \phi$. We have to show that there is $N>0$ such that for any $n>N, f^{n}(U) \cap V \neq \phi . h^{-1}(U)$ and $h^{-1}(V)$ are two $\theta$-open sets since $h$ is $\theta$-irresolute with $h^{-1}(V) \cap T \neq \phi$ and $h^{-1}(U) \cap T \neq \phi$. If the set $T$ is topologically $\lambda$-mixing then there is $N>0$ such that for any $n>M$, $g^{n}\left(h^{-1}(U)\right) \cap h^{-1}(V) \neq \phi$. So $\exists x \in g^{n}\left(h^{-1}(U)\right) \cap h^{-1}(V)$. That is $x \in g^{n}\left(h^{-1}(U)\right)$ and $x \in h^{-1}(V) \Leftrightarrow x=g^{n}(y)$ for $y \in h^{-1}(U) . h(x) \in V$. Thus, since $h \circ g^{n}=f^{n} \circ h$, so that $h(x)=h\left(g^{n}(y)\right)=f^{n}(h(y)) \in f^{n}(U)$ and we have $h(x) \in V$ that is $f^{n}(U) \cap V \neq \phi$. So, $h^{-1}(T)$ is $\theta$-mixing set.

Theorem 3.12 Let $(X, f)$ be a topological system and $A$ be a nonempty $\theta$-closed set of $X$. Then the following conditions are equivalent.

1) $A$ is a $\theta$-transitive set of $(X, f)$.

2) Let $V$ be a nonempty $\theta$-open subset of A and $\mathrm{U}$ be a nonempty $\theta$-open subset of $X$ with $U \cap A \neq \phi$. Then ther exists $n \in \mathbf{N}$ such that $V \cap f^{-n}(U) \neq \phi$.

3) Let $U$ be a nonempty $\theta$-open set of $\mathrm{X}$ with $U \cap A \neq \phi$. Then $\bigcup_{n \in \mathbf{N}} f^{-n}(U)$ is $\theta$-dense in $A$.

Theorem 3.13 Let $(X, f)$ be topological system and $A$ be a nonempty $\theta$-closed invariant set of $X$. Then $A$ is a $\theta$-type transitive set of $(X, f)$ if and only if $(A, f)$ is $\theta$-type transitive set.

\section{Proof:}

$\Rightarrow$ ) Let $V_{1}$ and $U_{1}$ be two nonempty $\theta$-open subsets of $A$. For a nonempty $\theta$-open subset $U_{1}$ of $A$, there exists a $\theta$-open set $U$ of $X$ such that $U_{1}=U \cap A$ Since $A$ is a $\theta$-type transitive set of $(X, f)$, there exists $n \in \mathbf{N}$ such that $f\left(V_{1}\right) \cap U \neq \phi$. Moreover, $A$ is invariant, i.e., $f(A) \subset A$, which implies that $f(A) \subset A$. Therefore, $f\left(V_{1}\right) \cap A \cap U \neq \phi$, i.e. $f\left(V_{1}\right) \cap U_{1} \neq \phi$. This shows that $(A, f)$ is $\theta$-type transitive.

$\Leftarrow)$ Let $V_{1}$ be a nonempty $\theta$-open set of $A$ and $U$ be a nonempty $\theta$-open set of $X$ with $A \cap U \neq \phi$, Since $U$ is an $\theta$-open set of $X$ and $A \cap U \neq \phi$, it follows that $U \cap A$ is a nonempty $\theta$-open set of $A$. Since $(A, f)$ is topologically $\theta$-type transitive, there exists $n \in \mathbf{N}$ such that $f\left(V_{1}\right) \cap(A \cap U) \neq \phi$, which implies that $f\left(V_{1}\right) \cap U \neq \phi$. This shows that $A$ is a $\theta$-type transitive set of 
$(X, f)$.

We now introduce four definitions of dense orbit and transitivity as follows:

Definition 3.14 Let $X$ be a topological space.

1) A map $f$ is said to have $\theta$-dense orbit if there exists $x \in X$ such that $C l_{\theta}\left(O_{f}(x)\right)=X$;

2) A map $f$ is said to have strictly dense orbit (resp., strictly $\theta$-dense orbit), if there exists $x \in X$ such that $C l\left(O_{f}(f(x))\right)=X$ (resp.

$\left.C l_{\theta}\left(O_{f}(f(x))\right)=X\right)$;

3) A map $f$ is said to be $\omega$-transitive if there exists $x \in X$ such that $\omega(x, f)=X$;

4) A map $f$ is said to be transitive (resp., $\theta$-transitive) if for any non-empty open (resp., $\theta$-open) sets $U$ and $V$ in $X$, there exists $n \in \mathbf{N}$ such that $f^{n}(U) \cap V \neq \phi$.

Now, we will discuss the relations between these orbits and transitivity maps in the theorem below:

Theorem 3.15

1) For any topological space $X$, each strictly $\theta$-dense orbit is a $\theta$-dense orbit, and each $\omega$-transitive map has strictly dense orbit and it is transitive.

2) For any topological space $X$, each continuous map which has strictly dense orbit is $\omega$-transitive and transitive.

\section{Acknowledgements}

First, thanks to my family for having the patience with me for having taking yet another challenge which decreases the amount of time I can spend with them. Specially, my wife who has taken a big part of that sacrifice, and also Sarmad, my son who helps me for typing my research. Thanks to all my colleagues for helping me for completing my research.

\section{References}

[1] Velicko, N.V. (1968) H-Closed Topological Spaces. (Russian) Mat. Sb. (N.S.) 70 (112) (1966), 98-112; English transl. American Mathematical Society Translations, 78, 102-118. https://doi.org/10.1090/trans2/078/05

[2] Dontchev, J. and Maki, H. (1998) Groups of $\theta$-Generalized Homeomorphisms and the Digital Line. Topology and Its Applications, 201-216.

[3] Jankovic, D.S. (1986) $\theta$-Regular Spaces. International Journal of Mathematics and Mathematical Sciences, 8615-8619.

[4] Kaki, M.N.M. (2015) Chaos: Exact, Mixing and Weakly Mixing Maps. Pure and Applied Mathematics Journal, Science PG, 4, 39-42.

[5] Kaki, M.N.M. (2015) New Concepts of Alpha-Chaotic Maps. Journal of Multidisciplinary Engineering Science and Technology (JMEST), 2, 187-190.

[6] Kaki, M.N.M. (2012) Introduction to $\theta$-Type Transitive Maps on Topological Spaces. International Journal of Basic \& Applied Sciences IJBAS-IJENS, 12, 104-108.

[7] Eie \& Chang (2010) A Course on Abstract Algebra. 144.

[8] https://en.wikipedia.org/wiki/Group_action\#cite_note-1 